\title{
Simulation Analysis of Different Thickness Control in the Continuous
}

\section{Hot-dip Galvanizing Process}

\author{
Zhang Hongmei ${ }^{1, a^{*}}$, Li Heran ${ }^{1, b}$, Fu Yujing ${ }^{2, \mathrm{c}}$ and Li Lianjie ${ }^{1, \mathrm{~d}}$ \\ ${ }^{1}$ University of Science and Technology Liaoning, Anshan Liaoning, China \\ ${ }^{2}$ Ben Xi Steel Group Corporation, Benxi Liaoning, China \\ a “lilyzhm68@126.com , b15938489@qq.com, 398575941@qq.com, 'dlj199108@163.com.
}

Keywords: Different thickness, Simulation analysis, Hot-dip galvanizing.

Abstract: The simulation of thickness control of different thickness galvanized steel sheet was researched in this paper, the simulation method featured by making use of bilateral calculation model to research Gas-jet wiping pressure of air knife. Regularity of the effect of steel strip and baffle gap on the thickness of edge coating is clearly found with the change of gas-jet wiping pressure and baffle gap. Simulation calculation method was used to calculate the value of surface pressure of the steel strip. Computation model of thickness was applied to compute surface coating thickness of strip.

\section{Introduction}

Galvanizing of steel substrate is one of the most commonly used methods to protect steels against the environment degradation [1]. Hot-dip galvanized coatings also have been used in industrial fields due to their excellent corrosion performance [2,3]. However, the application environment of a large number of galvanized steel sheets only has a high demand on the corrosion of single side of the steel strip, but to another side of the steel plain is relatively low. At this time, in order to reduce the costs, zinc layer thickness of one side of steel strip which corrosion performance is required relatively low should be controlled to cut down the zinc consumption [4,5]. Gas-jet wiping process is a hydrodynamic method to remove the excessive molten zinc adhered on the strip. In this process, a pair of opposing horizontal plane gas jets is located just above the bath, and the strip is vertically drawn up between the pair of opposing jets [6]. Controlling the coating thickness of galvanized steel strip bilateral precisely,which is a vital factor to reduce production costs. A simple and efficient method for controlling coating thickness is changing gas-jet wiping pressure of air knife[7,8]. The aim of this work is to control the coating thickness by altering gas-jet wiping pressure of air knife.

\section{Simulation Analysis}

\section{Models Created and Meshed}

Simulation process is based on the type $\mathrm{H}$ mode and three symmetric processings were adopted in the model. Thickness of the steel strip $t_{s}$ and thickness of the baffle $t_{\mathrm{b}}$ in the model is $0.5 \mathrm{~mm}$ and $2.5 \mathrm{~mm}$ respectively for the sake of making the symmetric processing more convenient. The value of height of air knife exit slit $\mathrm{d}$ is $1.1 \mathrm{~mm}$, the distance between exit slit of air knife and the steel plate $\mathrm{H}$ is adjusted by entity thickness which is on the freezing structure. The spacing between steel strip and the baffle $\mathrm{g}$ is $2 \mathrm{~mm}$, meanwhile in the symmetric processing side of air knife, outside surface 
of the model is printed space along the direction by the length of $0.55 \mathrm{~mm}$.

"Sweep" method was used to mesh every body of the model, we set steel strip and baffle as wall surface and define steel strip and baffle as relative slip wall boundary condition and no-slip wall boundary condition respectively. The rest of boundaries were defined as pressure outlet boundary condition, among which the outlet pressure value is the atmospheric pressure.

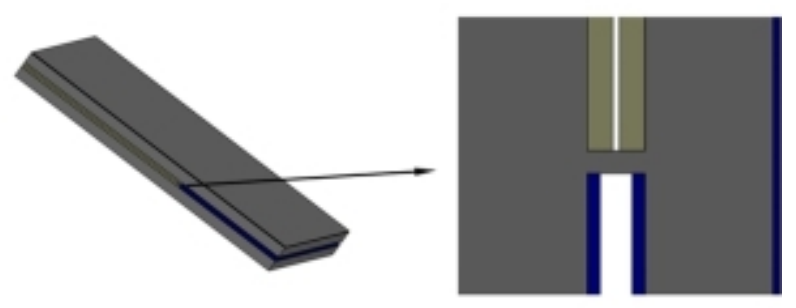

Fig. 1 Simplified calculation model.

\section{Setting of the Working Conditions}

Different injection pressures were used in this research, relative property settings of model depended on different working conditions. The relevant parameters in the model are shown in table.1.

Table 1 Air knife and strip process parameters

\begin{tabular}{lll}
\hline Name & Parameter & Value \\
\hline \multirow{4}{*}{ Air knife } & Outlet pressure $P_{0}[\mathrm{kPa}]$ & $10,11,12,13,14,15$ \\
& $\begin{array}{l}\text { Distance between injector } \\
\text { and steel strip } H[\mathrm{~mm}]\end{array}$ & 10,15 \\
& Opening-degree $d[\mathrm{~mm}]$ & 1.1 \\
Speed $v_{s}\left[\mathrm{~m} \cdot \mathrm{s}^{-1}\right]$ & \\
Steel strip & Width B $[\mathrm{mm}]$ & 160 \\
& Thickness $t_{s}[\mathrm{~mm}]$ & 0.5 \\
Baffle & Baffle thickness $t_{b}[\mathrm{~mm}]$ & 2.5 \\
\hline
\end{tabular}

\section{Results and discussions}

The evolution of pressure contours of the air knife jetting side as different injection pressures change is shown in fig. 2, the surface pressure of the strip has been changed obviously when the injection pressure of air knife was transformed. The surface pressure of steel strip which injection pressure is $11 \mathrm{Kpa}$ is significantly higher than that which is $10 \mathrm{Kpa}$. The airflow vortex should have emerged in the gap which has deviated towards the side of less pressure due to the raise of the unilateral injection pressure in the gap of baffle and the steel strip. 


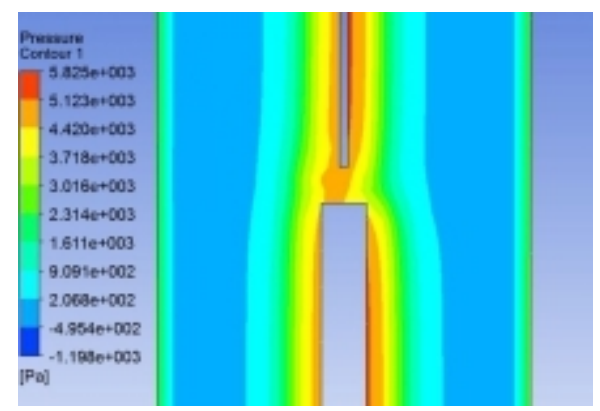

$10 \mathrm{kPa}-11 \mathrm{kPa}$

Fig. 2 The strip surface pressure distribution under different injection pressure.

With the raise of unilateral injection pressure, the overall pressure of strip surface of the side which pressure increased was enhanced and the airflow vortex in the gap of air knife and baffle deviated towards the side which injection pressure is weak which results in the increase of strip edge part pressure of the side where injection pressure is weak. A large number of simulations indicate that airflow vortex will deviate from gap zone due to pressure difference continue to increase, and the airflow vortex will move forward towards the side which injection pressure is weak as shown in fig. 3 .

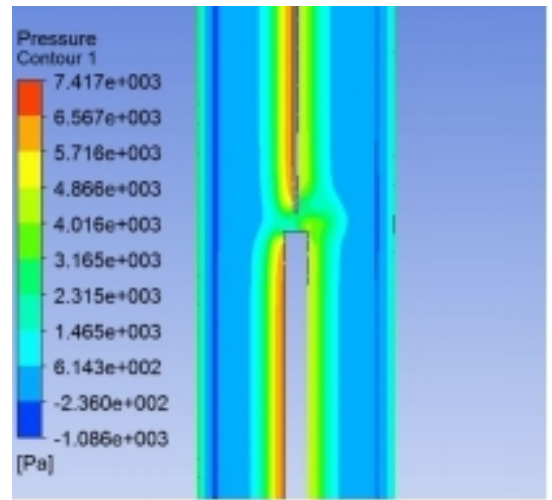

Fig. 3 The strip surface pressure distribution under unilateral injection pressure

$$
13 \mathrm{kPa} \text {. }
$$

The pressure value of strip surface center and edge part is $4.93 \mathrm{kPa}$ and $4.81 \mathrm{kPa}$ respectively as shown in fig. 4. The pressures of steel strip surface change with the improvement of the unilateral injection pressure, when the injection pressure increase by $1 \mathrm{kPa}$, the surface pressure of the strip no matter the center or the edge is improved by $500 \mathrm{~Pa}$.

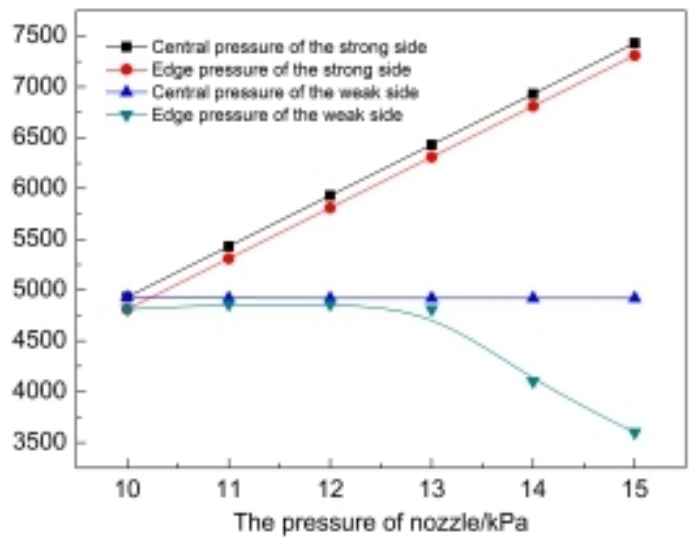

Fig. 4 When the double gas injection pressure is different the distribution of strip surface pressure. 
The thickness calculation of the strip surface coating is based on the injection pressure, and the distribution of coating of the strip surface is base on the coating thickness calculation model are shown in fig. 5.

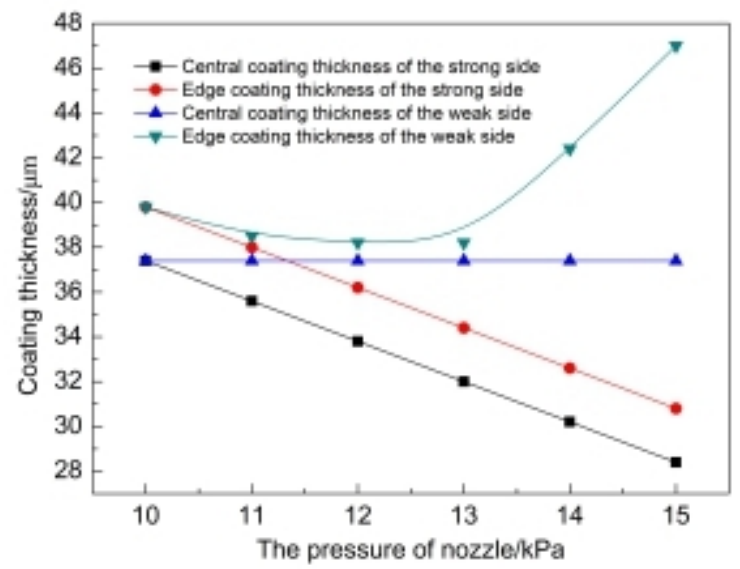

Fig. 5 Distribution of coating under different injecting pressure.

\section{Conclusions}

(1) The airflow in the gap between the steel strip and baffle moves toward the side that gas-jet wiping pressure is weak which led to the pressure enhancement of the strip edge part.

(2) With the increase of unilateral pressure by $1 \mathrm{kPa}$, the thickness of the coating decreased by $1.8 \mu \mathrm{m}$. The gas-jet wiping pressure difference of the air knife should not be more than $2 \mathrm{kpa}$ when different injection pressures are applied to control difference thickness.

\section{References}

[1] A.R.Marder, Prog. Mater. Sci. 45(2000) 191.

[2] M.Zapponi, A. Quiroga and T. Perez: Surf. Coat. Technol.,122(1999), 18.

[3] Hamed ASGARI, Mohammad Reza TOROGHINEJAD and Mohammad Ali GOLOZAR. The Role of Texture and Microstructure in Optimizing the Corrosion Behavior of Zinc Hot-dip coated Steel Sheets.

[4] ASTM. Standard Specification for Steel Sheet, Zinc-Coated (Galvanized) or Alloy-Coated by the Hot-Dip Process [M]. U.SA.: 2009, 1-13.

[5] Miller.CA, Neogi.P, Interfacial Phenomena[J], Dispersion Science and Technology, 1986,7(1): 127-128.

[6] Hongyun SO, Hyun Gi YOON, Myung Kyoon CHUNG. CFD Analysis of Sag Line Formation on the Zinc-coated Steel Strip after the Gas-jet Wiping in the Continuous Hot-dip Galvanizing Process.ISIJ International, Vol. 51(2011).

[7] Gagne.M. Hot dip galvanizing with Zinc-Bismuth Alloy.Metall,1999,53(5),269-271.

[8] D. LACANETTE, S. VINCENT, E. ARQUIS, et al. Numerical Simulation of Gas-Jet Wiping in Steel Strip Galvanizing Process[J].ISIJ International,2005, 45(2):214-220. 